\title{
Asian dobsonflies (Megaloptera: Corydalidae) of the collection of Zoological Institute of Russian Academy of Science, Saint Petersburg
}

\author{
Азиатские коридалы (Megaloptera: Corydalidae) комлекщии \\ зоологического института РАН, Санкт-Петербург
}

\author{
Xingyue Liu', Victor Krivokhatsky ${ }^{2}$, Julia V. Samartseva ${ }^{2}$ \\ С. $\Lambda$ ью ${ }^{1}$, В.А. Кривохатский ${ }^{2}$ Ю.В. Самарцева ${ }^{2}$
}

\footnotetext{
${ }^{1}$ Department of Entomology, China Agricultural University, Beijing, 100193, China. E-mail: xingyue_liu@yahoo.com.

2 Zoological Institute, Russian Academy of Sciences, Universitetskaya nab. 1, 199034, Saint Petersburg, Russia. E-mail: myr@pochta.ru.

2 Зоологический институт РАН, Университетская наб. 1, Санкт-Петербург 199034, Россия.
}

KEY WORDS. Corydalinae, Chauliodinae, dobsonflies, Asia, new records.

КЛЮЧЕВЫЕ СЛОВА. Corydalinae, Chauliodinae, коридалы, Азия, новые находки.

ABSTRACT. The annotated list of 32 species of dobsonflies of subfamilies Corydalinae and Chauliodinae of Eastern Palearctic and Oriental Region from collection of Zoological Institute of Russian Academy of Science is given. Three species, Neoneuromus ignobilis Navás, 1932, Protohermes yunnanensis Yang et Yang, 1988 and Neochauliodes punctatolosus Liu et Yang, 2006, which were earlier known from southern China and northern Vietnam, are recorded in the southern Vietnam for the first time. Moreover, the female of Nevromus testaceus Rambur, 1842 earlier known as the Javanese and Sumatran endemic was caught in March 1995 by A.V. Gorokhov in Vietnam. This record is associated with the random drift of the specimen by the tsunami which was occurred in 1995 in Indonesia. Another specimen of Protohermes sp. from northern Vietnam is the new species and will be describe by Dr. Liu in the separate publication.

РЕЗЮМЕ. В работе приводится аннотированный список 32 вида коридалов подсемейств Corydalinae и Chauliodinae фауны Восточной Палеарктики и Ориентального царства из коллекции Зоологического института РАН. Три вида, ранее известные из южного Китая и северного Вьетнама, Neoneuromus ignobilis Navás, 1932, Protohermes yunnanensis Yang et Yang, 1988 и Neochauliodes punctatolosus Liu et Yang, 2006, теперь, по материалам коллекции зарегистрированы из южного Вьетнама. Кроме того, одна самка Nevromus testaceus Rambur, 1842, до сих пор известная как эндемик Суматры и Явы, была поймана А.В. Гороховым в марте 1995 г. во Вьетнаме. Эта поимка связывается с заносом этого экземпляра цунами, документально произошедшим в 1995 г. в Индонезии. Ещё один коллекционный экземпляр из рода Protohermes из Северного Вьетнама оказался новым видом, подготовленным к описанию д-ром Лью, и войдет в типовую серию.

\section{Preface}

The holometabolan order Megaloptera belongs to the superorder Neuropterida and comprises only two extant families, i.e. Corydalidae and Sialidae, currently with ca. 380 species in the world [Yang, Liu, 2010; Liu et al., 2015]. The larvae of Megaloptera are exclusively aquatic. However, the megalopteran adults, especially those of Corydalidae, are diagnostic insects, some of which are remarkably large-sized with enlarged mandibles, and they can be usually collected by light trap near the aquatic habitats. The collection of Megaloptera in the Institute (recently Zoological Museum) originated from the specimens, purchased from insect trader Staudinger and rather than from own Russian zoologists. More productive collectors of dobsonflies in Asian countries were A.V. Abramov, V.M. Gnezdilov, A.V. Gorokhov, S.A. Ryabov, V.A. Trjapitzyn, N.N. Vinokurov, A.K. Zagulyaev.

V.L. Bianchi, O.M. Martynova, S.G. Lepneva, L.A. Zhiltzova were curators of the collection in different periods. Victor Krivokhatsky — is the last curator, and Julia Samartseva - is a keeper of collection.

About 6 genera from the New World and 2 genera from Australia are presented in the Institute collection, but not type material. One Russian species of Corydalidae, Megaloptera, was in interest of T.S. Vshivkova. She later got types as a loan after first description [Vshivkova, 1995] but has not yet returned the loaned material. Some photos from these specimens were published in her last publication [Vshivkova, Dubatolov, 2010)].

The present list contains 32 Asian species of Corydalidae including both subfamilies Corydalinae and Chauliodinae, which are considerably diverse with more than 150 species in the Asian part of Palaearctic and Oriental region. Most specimens of all species listed here belong to the known areas of distribution reported in Yang, Liu 
[2010], Liu et al. [2006, 2009, 2012] and Letardi et al. [2012]. However, three species, i.e. Neoneuromus ignobilis Navás, 1932, Protohermes yunnanensis Yang et Yang, 1988 and Neochauliodes punctatolosus Liu et Yang, 2006, which were known in southern China and northern Indochina, are first recorded in southern Vietnam. In addition, a single female of Nevromus testaceus Rambur, 1842, which was known previously as endemic to Sumatra and Java, was collected in Vietnam by A.V. Gorokhov in March of 1995. That capture could be connected with specimen transfer with tsunami, occur in 1995 in Indonesia. Next one specimen of Protohermes from Northern Vietnam is an unpublished new species.

\section{Systematic part}

\section{Corydalidae \\ Corydalinae}

Acanthacorydalis orientalis (McLachlan, 1899)

MATERIAL. 19, China, Sichuan, $60 \mathrm{~km} \mathrm{NW}$ of Chengdu, Matsaonin vil., the experimental station, $31.269161^{\circ} \mathrm{N}$, $103.323669^{\circ} \mathrm{E}$, summer 1954 , anonym coll.

Acanthacorydalis fruhstorferi van der Weele, 1907

MATERIAL. 19 , Northern Vietnam, Lao Cai Prov., $6 \mathrm{~km} \mathrm{~W}$ of $\mathrm{Sa} \mathrm{Pa}$, north slope of Phansipan Mt. area, near Tram Ton (station of

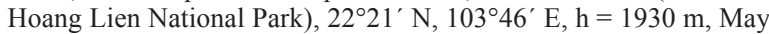
2008, A.V. Abramov coll.

Neoneuromus ignobilis Navás, 1932

MATERIAL. 20', China, Yunnan, Tszindun [Xinping Yi and Dai Autonomous County], 26-28.05.1956, A.K. Zagulyaev coll.;
10', Vietnam, Son La Prov., Son La env., Na Ng'u; at light, 10.05.1986, V.A. Trjapitzyn coll.; 10 $0^{\top}$, Amci Thau, Neuromus fenestralis [McL., $\sigma^{7}$, coll. Staudinger]; 1우, Vietnam, Lam Dong Prov., Bidoup-Nui Ba Nat. Park, 5 km NE Long Lanh Vill., Giang Ly, h $1444 \mathrm{~m}, \mathrm{~N} 12^{\circ} 10.960^{\prime} \mathrm{E} 108^{\circ} 40.792^{\prime}$, at light, 23-27.V.2014, V.M. Gnezdilov coll.

DISTRIBUTIONAL NOTES. New record for southern Vietnam (Figs 1-2).

Neoneuromus maclachlani (van der Weele, 1907)

MATERIAL. 10, N. Vietnam, Tam Dao, Vinh Phuc Prov., h= 800-900 m, 12.06.1994, A. Baranov coll.; 10 $0^{7}$, N. Vietnam, Tam Dao, Vinh Phuc Prov., h = 800-900 m, 3.07.1994, A. Monastyrskyi coll.; $20^{\top}, 1$,, N. Vietnam, Tam Dao, Vinh Phuc Prov., h $=800-900$ m, 17.05.1995, A.V. Gorokhov coll.; 1ㅇ, N. Vietnam, Tam Dao, 9.06.1997, S.A. Ryabov coll.; 10', Omei Shan [China; Sichuan Prov.], Chauliodes sinensis Walk., anonym coll. and det.

Neoneuromus orientalis Liu et Yang, 2004

MATERIAL. 2q, Vietnam, no other locality.

Neoneuromus tonkinensis (van der Weele, 1907)

MATERIAL. 20, N. Vietnam, Tam Dao, Vinh Phuc Prov, $\mathrm{h}=$ 800-900 m, 17-31.05.1995, A.V. Gorokhov coll.; 1ㅇ, N. Vietnam, Tam Dao, Vinh Phuc Prov., $h=800-900$ m, 17.05.1995, A.V. Gorokhov coll.

Neurhermes maculipennis (Gray, 1832)

MATERIAL. $2 \sigma^{\top}, 2+$, Ases, Chirique Neuromus ruficollis Gerst., coll. Staudinger.

\section{Nevromus exterior (Navás, 1927)}

MATERIAL. 29, Vietnam, Son La Prov., Son La vill., 3.05.1986, A. V. Gorokhov coll.; 1 9 , Vietnam, Son La Prov., Son La env., Na Ng'u, at light, 12.05.1986, V.A. Trjapitzyn coll.; 8, Vietnam, Gia Lai Prov., 20 km N of Kannak v., Buon Loi, 22-31.03.1995, A.V. Gorokhov coll.

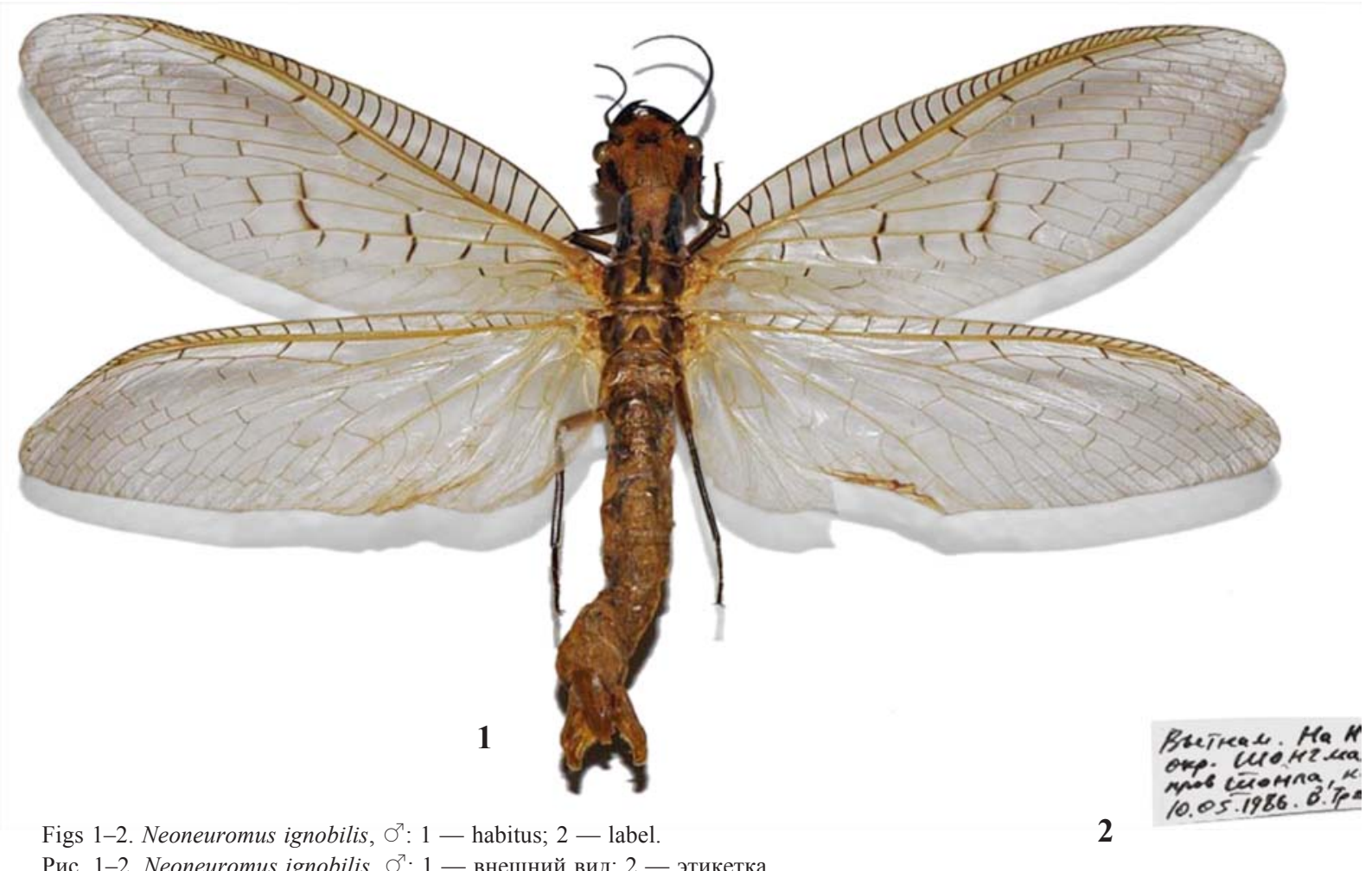

Рис. 1-2. Neoneuromus ignobilis, $\sigma^{\top}: 1$ - внешний вид; 2 - этикетка. 
Nevromus testaceus Rambur, 1842

MATERIAL. 19, Vietnam, Gia Lai Prov., $20 \mathrm{~km} \mathrm{~N}$ of Kannak v., Buon Loi, 22-31.03.1995, A.V. Gorokhov coll.

DISTRIBUTIONAL NOTES. This species was known as endemic to Sumatra and Java. So, that is the first record for Vietnam. The specimen has broken costal area of forewing (Figs 3-4), which could be aftermath of the typhoon, usually having in the region meridional direction, from Indonesia to Vietnam.

Protohermes albipennis (Walker, 1853)

MATERIAL. 1, Nepal, 32 km E Kathmandu, Nagarkot, hotel, 21.05.2012, at light, A. Bezborodkin coll.

Protohermes axillatus Navas, 1932

MATERIAL. 2 9 , Northern Vietnam, Lao Cai Prov., $6 \mathrm{~km} \mathrm{~W}$ of $\mathrm{Sa} \mathrm{Pa}$, north slope of Phansipan Mt. area, near Tram Ton (station of Hoang Lien National Park), $22^{\circ} 21^{\prime} \mathrm{N}, 103^{\circ} 46^{\prime} \mathrm{N}, \mathrm{h}=1930 \mathrm{~m}$, May 2008, A.V. Abramov coll. (Figs 5-6).

Protohermes davidi van der Weele, 1909

MATERIAL. 107, China, C Sichuan, Dayi distr., Chadiping env., 1200-1500 m, 5-7.08.1996, A.I. Miroshnikov \& A. Zamotajlov coll.; 19, China, Sichuan, Lunan'fu, Hodzigou, $6000 \mathrm{ft}$, $07-$ 08.93, Berezov coll

Protohermes differentialis (Yang et Yang, 1986)

MATERIAL. $20^{\top}$, N. Vietnam, Tam Dao, Vinh Phuc Prov., h= 1000 m, 9-11.04.1996, A. Gorokhov coll.; 1오, N. Vietnam, Tam Dao, Vinh Phuc Prov., 12.06.1994, A. Baranov coll.

Protohermes grandis (Thunberg, 1781)

(catalog): 1 sp. — № 776, coll. Staudinger.

MATERIAL. $10^{7}$, Neurimus grandis Thunb.; Japan. Kotljarevskyi coll.; 1우, Neurimus grandis Thb.; Japan, № 776, coll. Staudinger.; 1+, Japan, Kyushu, Obama; 8.VIII.1907; Cherskyi coll.; 1오. Hokkaido otoineppi; 30-31.VII.1997; N.N. Vinokurov coll.

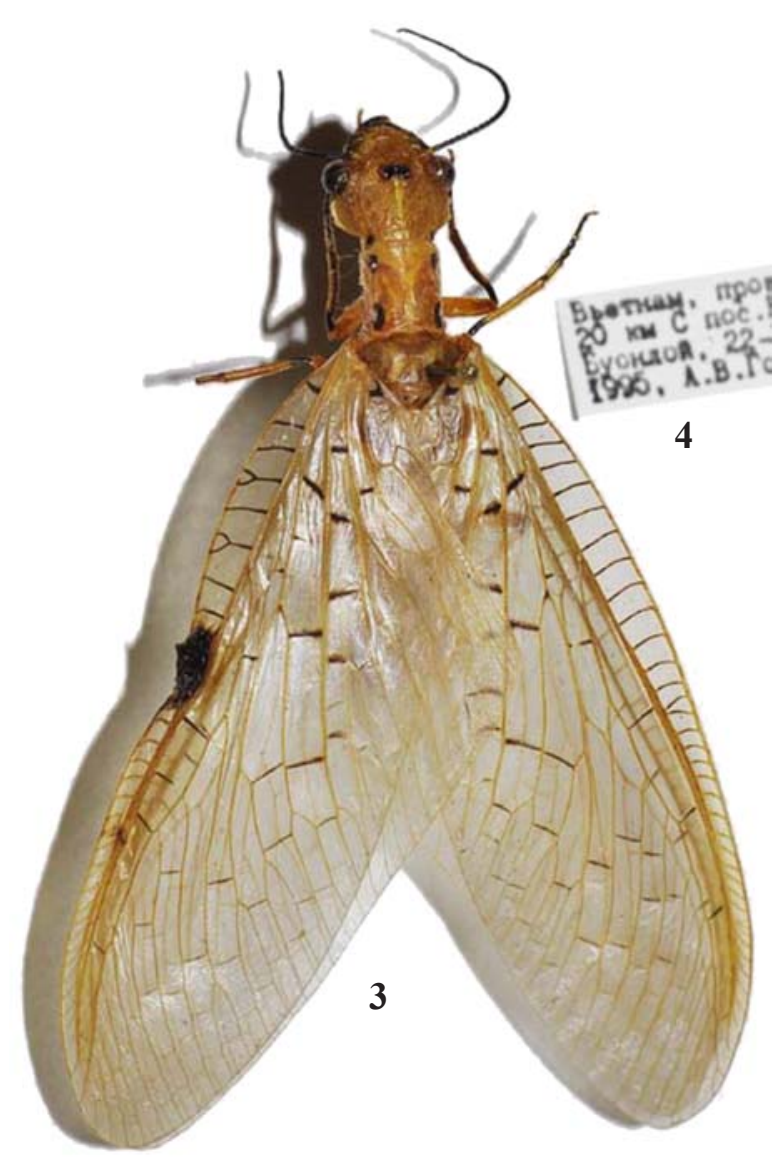

Figs 3-4. Nevromus testaceus, : : 5 - habitus; 6 - label.

Рис. 3-4. Nevromus testaceus, этикетка.
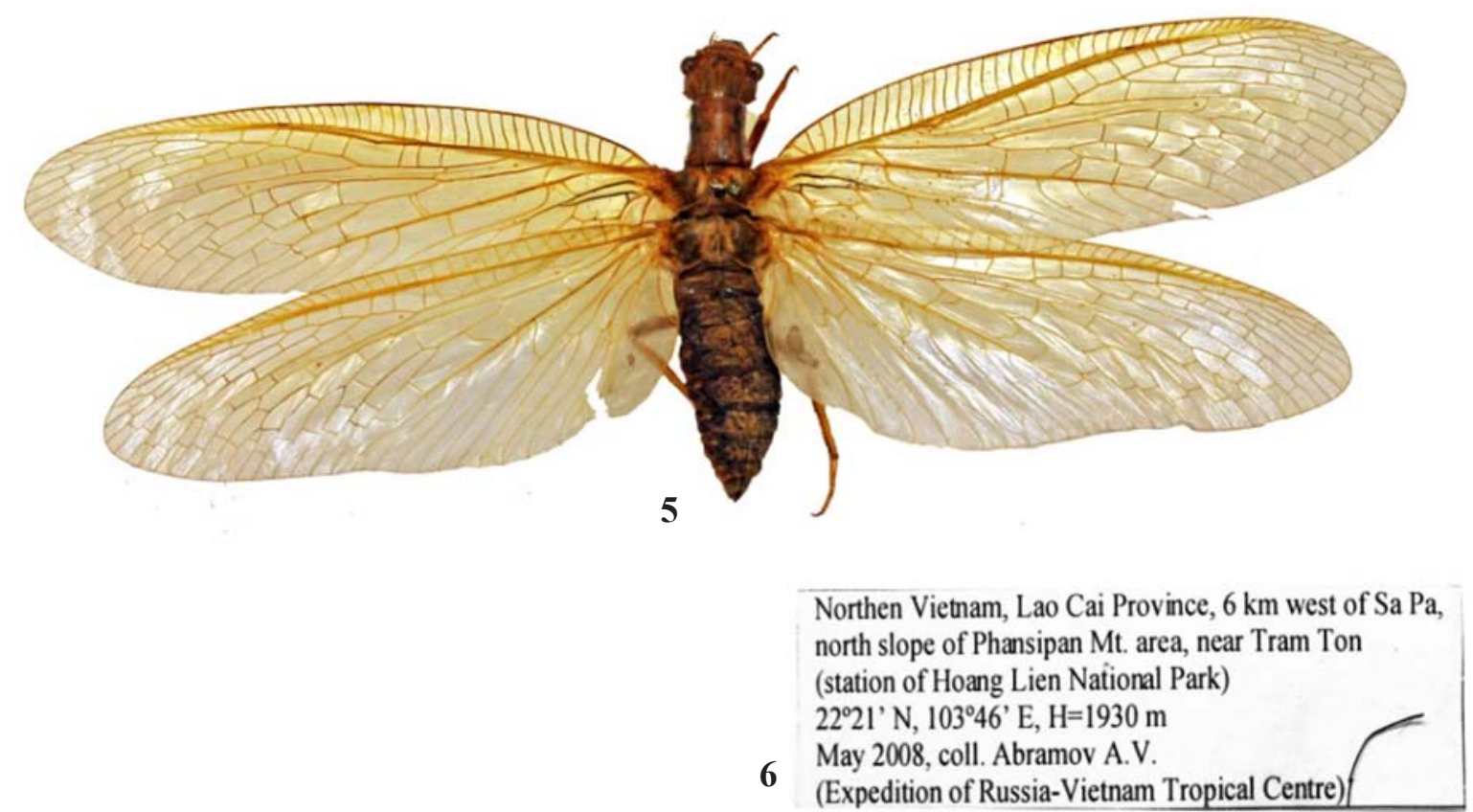

Figs 5-6. Protohermes axillatus, 9 : 5 - habitus; 6 - label.

Pис 5-6. Protohermes axillatus, + : 5 - внешний вид; 6 - этикетка. 
Protohermes guangxiensis Yang et Yang, 1986

MATERIAL. 19, N. Vietnam, Tam Dao, Vinh Phuc Prov., 3.07.994, A. Monastyrskyi coll.; 19, N. Vietnam Tam Dao, 14.06.01, S. Ryabov coll.

Protohermes sinuolatus Liu, Hayashi et Yang, 2009

MATERIAL. 19, Vietnam, Gia Lai Prov., 20 km N of Kannak v., Buon Loi, 22-31.03.1995, A.V. Gorokhov coll.

Protohermes striatulus Navás, 1926

MATERIAL. $2 \sigma^{\top}, 3 \circ$, N. Vietnam, Tam Dao, Vinh Phuc Prov., $\mathrm{h}=800-900 \mathrm{~m}, 17-31.05 .1995$, A.V. Gorokhov coll.

Protohermes xanthodes Navás, 1913

MATERIAL. 3ㅇ, N. China, Tianjin, 9.VII.1914, Yu. Vasiliev coll.; SYNTYPES Protohermes martynovae Vshivkova, 1995 [defined in Vshivkova, Dubatolov, 2010]: 10 1 , 1, Russia, Jakovlevka Village, Spasskyi Uezd, Ussurisky Region [Primorsky Region], 17.VII.1926, col. A.M. Djakonov, N.N. Filipjev (dry, pinned material replaced in alcohol); $10^{7}$, Kvashuk's Honey Farm at Jakovlevka Village, Spasskyi Uezd, Ussurisky Region, 28.VI.1926, col. A.M Djakonov,N.N. Filipjev (dry, pinned material) (reported in Vshivkova, Dubatolov, 2010 as deposited in Zoological Institute of RAN, St-Petersburg, but still does not returned after the loan).

SYSTEMATICAL NOTES. Protohermes matynovae Vshivkova, 1995 was synonymized with Protohermes xanthodes by Liu et al. [2006]. Then P. martynovae is treated as the junior synonym of $P$. xanthodes in LDL and sequences publications. There is no doubt they are same species although Vshivkova still prefers to the validity of $P$. martynovae. More over the name $P$. martynovae is nomen nudium because placed in interrupted description: Vshivkova, 1995 - is only key, and Vshivkova, Dubatolov, 2010 - redescription and types designation.

ICZN: 10.1.1. If publication of the data relating to a new nominal taxon or a nomenclatural act is interrupted and continued at a later date, the name or act becomes available only when the requirements of the relevant Articles have been met.
Protohermes yunnanensis Yang et Yang, 1988

MATERIAL. 1으, Vietnam, Lam Dong Prov., Bidoup-Nui Ba Nat. Park, $5 \mathrm{~km}$ NE Long Lanh Vill., Giang Ly, h $1444 \mathrm{~m}$, $\mathrm{N} 12^{\circ} 10.960^{\prime} \mathrm{E} 108^{\circ} 40.792^{\prime}$, at light, 23-27.V.2014, V.M. Gnezdilov coll. Vietnam.

DISTRIBUTIONAL NOTES. New record for southern

\section{Protohermes sp.}

MATERIAL. $10^{\top}$, N. Vietnam, Lao Cai Prov., $6 \mathrm{~km} \mathrm{~W}$ of Sapa, $\mathrm{h}=1950 \mathrm{~m}, 05.2008$, A.V. Abramov coll.

NOTES. This is an unpublished new species previously found by Xingyue Liu. This will be a paratype since this new species is described.

\section{Chauliodinae}

Ctenochauliodes abnormis Yang et Yang, 1986

MATERIAL. $10^{7}, 1$,, N. Vietnam, Tam Dao, Vinh Phuc Prov., $\mathrm{h}=800-900 \mathrm{~m}, 17-31.05 .1995$, A.V. Gorokhov coll.; 1\%, Vietnam, Hoa Binh Prov., Mai Chau Distr., Pa Co, 2044’ N, 10455'E, 1120 m, 22-24.04.2002, S. Belokobylskij coll.

Ctenochauliodes nigrovenosus (van der Weele, 1907)

MATERIAL. 1 , , N. Vietnam, Cao Bang Prov., Nguen Binh, h = 800 m, 1-15.05.03, S.A. Ryabov coll.

Neochauliodes bicuspidatus Liu et Yang, 2006

MATERIAL. 19 , N. Vietnam, Lao Cai, $6 \mathrm{~km} \mathrm{~W}$ of Sapa, h = 1950 m, 05.2008, A.V. Abramov coll.

Neochauliodes formosanus (Okamoto, 1910)

MATERIAL. 10', China, Foochow, May 30 1957, M.S. Yang

Neochauliodes koreanus van der Weele, 1909

MATERIAL. 407, 4, N. Vietnam, Tam Dao, Vinh Phuc Prov., $\mathrm{h}=800-900 \mathrm{~m}, 17-31.05 .1995$, A.V. Gorokhov coll.

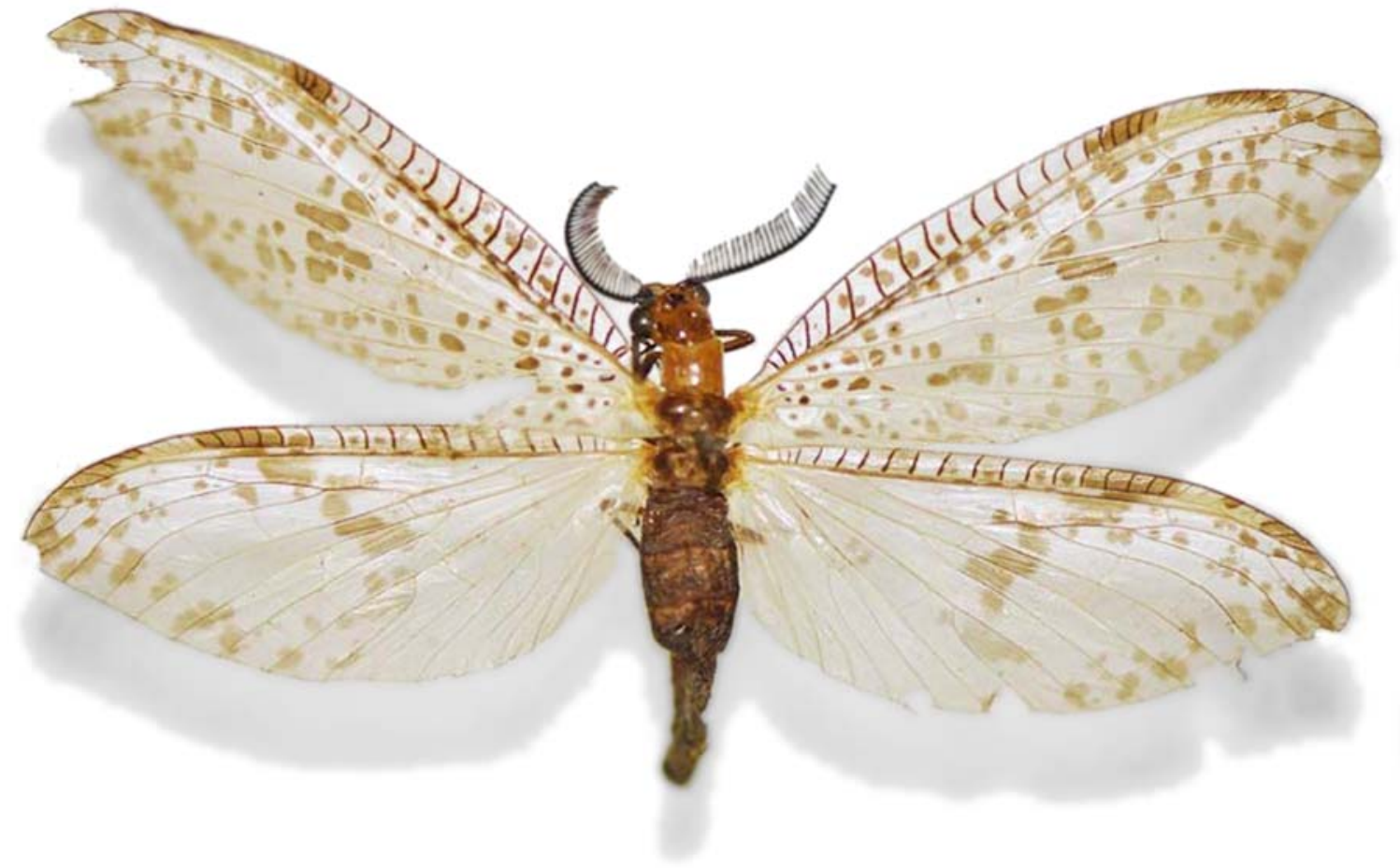

Fig. 7. Neochauliodes punctatolosus: O', Vietnam, Son La Prov., Son La env., Na Ng'u; at light, 4.05.1986 V.A. Trjapitzyn coll. Рис. 7. Neochauliodes punctatolosus: О', Вьетнам, провинция Шон Ла, окр. Шон Ла, На Нгу, на свет 4.05.1986 В.А. Тряпицын. 
Neochauliodes meridionalis van der Weele, 1909

MATERIAL. 10', Vietnam, Ha Tinh Prov., Son Kim, 112.04.2003 S.A. Ryabov coll.

Neochauliodes moriutii Asahina, 1987

MATERIAL. $10^{\Upsilon}$, N. Thailand, Lampang prov., Buan Pa Mieng, 1.08.2011, A. Pushenkov coll.

Neochauliodes punctatolosus Liu et Yang, 2006

MATERIAL. 107, China, Yunnan, Tszindun [Xinping Yi and Dai Autonomous County]; Dun- Dzja-fui Mt., 21.06.1956, A. Zagulyaev coll.; $10^{\prime}$, China, Yunnan, Tszindun [Xinping Yi and Da Autonomous County], 12.06.1956, A. Zagulyaev coll.; $10^{7}$, Vietnam, Son La Prov., Son La env., Na Ng'u; at light, 4.05.1986, V. Trjapitzyn coll.; $10^{\top}$, Vietnam, Lam Dong Prov., Bidoup-Nui Ba Nat. Park, $5 \mathrm{~km}$ NE Long Lanh Vill., Giang Ly, h $1444 \mathrm{~m}$, N12 ${ }^{\circ} 10.960^{\prime}$ E10840.792', At light, 23-27.V.2014, V.M. Gnezdilov coll.

DISTRIBUTIONAL NOTES. New record for southern Vietnam (Fig. 7).

Neochauliodes tamdaoensis Liu, Hayashi et Yang, 2010

MATERIAL. $10^{7}$, N. Vietnam, Tam Dao, Vinh Phuc Prov., h = 800-900 m, 17-31.05.1995, A. Gorokhov coll.

Neochauliodes tonkinensis (van der Weele, 1907)

MATERIAL. 10', China, Sichuan, Yundian, $132 \mathrm{~km} \mathrm{H-Way}$ Dali-Kunming, $\mathrm{h}=1618 \mathrm{~m}, 10.06 .2002$, M. Volkovich coll.; $20^{7}$, China, Yunnan, Tszindun [Xinping Yi and Dai Autonomous County], 1.06.1956, A. Zagulyaev coll.; 10", 1ㅇ, N. Vietnam, Tam Dao, Vinh Phuc Prov., h $=800-900 \mathrm{~m}, 17-31.05 .1995$, A.V. Gorokhov coll.; 2ㅇ, Vietnam, Lao Cai Prov., Sa Pa Distr., Fan Si Pan Mt., $22^{\circ} 21^{\prime} \mathrm{N}, 103^{\circ} 46^{\prime} \mathrm{E}, 2000 \mathrm{~m}, 20.04-9.05 .1999$, N.L. Orlov coll.; $30^{7}, 1$, , Vietnam, Lao Cai Prov., $6 \mathrm{~km} \mathrm{~W}$ of Sa Pa, h $=1950 \mathrm{~m}$, 05.2008, A.V. Abramov coll.; 19, Burma, Carin Cheba, 900-1100 m, VI.1988, L. Fea coll.

Neochauliodes umbratus Kimmins, 1954

MATERIAL. $10^{7}, 1$,, N. Vietnam, Tam Dao, Vinh Phuc Prov. $\mathrm{h}=800-900 \mathrm{~m}, 9-11.04 .1986$, A.V. Gorokhov coll.; 1 우, N. Vietnam, Tam Dao, Vinh Phuc Prov., h $=800-900$ m, 08.05.1994, A.V. Gorokhov coll.; 2 ㅇ, N. Vietnam, Tam Dao, Vinh Phuc Prov., h =
800-900 m, 17.05.1995, A.V. Gorokhov coll.; $2 \sigma^{7}, 1$ 오 N. Vietnam, Tam Dao, Vinh Phuc Prov., h = 800-900 m, 12.06.1994, A. Baranov coll.

Parachauliodes asahinai Liu, Hayashi \& Yang, 2008

MATERIAL. 1 ex. (Sex unknown), Japan, Kotljarevskyi coll.; Chauliodes japonicus Mc. Lh. anonym det.

Parachauliodes continentalis van der Weele, 1909

MATERIAL. 10', 1ㅇ, Chauliodes japonicus M.L., Japan, coll. Staudinger.

\section{References}

International Commission on Zoological Nomenclature. International Code of Zoological Nomenclature online. 4-th edition. 2012. http://iczn.org/iczn/index.jsp.

Letardi A., Hayashi F., Liu X.Y. 2012. Notes on some dobsonflies and fishflies (Megaloptera: Corydalidae) from northern Vietnam // Entomotaxonomia. Vol.34. No.4. P.641-650.

Liu X.Y., Hayashi F., Yang D. 2006. Systematics of the Protohermes xanthodes species-group in eastern Asia (Megaloptera: Corydalidae) // Entomological Science. Vol.9. P.399-409.

Liu X.Y., Hayashi F., Yang D. 2009. Notes on the genus Protohermes van der Weele (Megaloptera: Corydalidae) from Vietnam, with description of two new species // Zootaxa. Vol.2146. P.22-34.

Liu X.Y., Hayashi F., Viraktamath C.A., Yang D. 2012. Systematics and biogeography of the dobsonfly genus Nevromus Rambur (Megaloptera: Corydalidae: Corydalinae) from the Oriental realm // Systematic Entomology. Vol.37. P.657-669.

Liu X.Y., Lü Y.N., Aspöck H., Yang D., Aspöck U. 2015. Homology of the genital sclerites of Megaloptera (Insecta: Neuropterida) and their phylogenetic relevance // Systematic Entomology. DOI: $10.1111 /$ syen.12154.

Yang D., Liu X.Y. 2010. Fauna Sinica Insecta. Vol.51. Megaloptera. Science Press, Beijing. 457 pp, XIV pl. [in Chinese].

Vshivkova T.S. 1995. [Order 32. Megaloptera // P.A. Lehr (ed.). Key for Insects of the Far East of Russia]. Vol.4. No.1. P.10-34 [in Russian]

Vshivkova T.S., Dubatolov V.V. 2010. New records of Protohermes species (Megaloptera, Corydalidae, Corydalinae) from the south of the Russian Far East // Euroasian Entomological Journal. Vol.9. No.3. P.345-351. 